\title{
Analytical Software for Operating With a Set of real and Virtual Objects Using the Rules of Cartesian Closed Category
}

\author{
Gennadiy Kulikov \\ Ufa State Aviation Technical University \\ Ufa, Russia \\ gennadyg_98@yahoo.com
}

Maria Shilina

Ufa State Aviation Technical University

Ufa, Russia

lurik@mail.ru

\author{
Vyacheslav Antonov \\ Ufa State Aviation Technical University \\ Ufa, Russia \\ antonov.V@bashkortostan.ru
}

Lyudmila Rodionova

Ufa State Aviation Technical University

Ufa, Russia

maria.shilina@gmail.com

\author{
Almira Fakhrullina \\ Ufa State Aviation Technical \\ University \\ Ufa, Russia \\ almirafax@mail.ru
}

\begin{abstract}
In the paper the problem of formalization and identification of real and virtual information objects and traceability of their relations in the studied subject domain using the rules of monomorphism and polymorphism of the mathematical theory of sets and categories is investigated. According to the principles of the systems engineering standard ISO/IEC 15288 models and methods of analytical software creation are developed. It is shown that the structure of such analytical software meets the conditions of Cartesian closed logic that considerably expands the range of tasks, which can be solved using this software. This is made possible because the effective reengineering of the software can be carried out in real time mode without any changes of its program code. The formalized description of the relations between virtual and real objects using the rules of data storages is offered. The method of creation of Chomsky hierarchies and a method of Osgood's semantic differentials are applied. As an example the structure of the distributed analytical software of the talent pool management of the industrial enterprise formed together with educational institutions of the higher education is considered.
\end{abstract}

Keywords-analytical software, systems engineering, the principle of semantic transformation and semantic differentials, the theory of sets categories, real and virtual objects, Cartesian closed category.

\section{INTRODUCTION}

The research is based on the following papers: in the field of the theory and mathematical design methods of large-scale information systems on the works of Kovalev S.P. [1]; in the field of fractal approach to knowledge structuring, Intelligent systems and decision-making support systems on the works of Massel L.V. [2]; in the field of interaction and self-organization modeling in modern interfaces, networks and environments and also software and mathematical modeling of integration processes of diverse data, system engineering and development on the works of Beltukov A.P., Kulikov G.G., Antonov V.V. [3-6]; in the field of tasks of intelligent management of educational processes at the higher school on the works of Jussupova N.I. [7]; in the field of modeling and methods of quality management on the basis of applications of artificial intelligence on the works of Kovács G.L. [8]; in the field of topos and the category theory on the works of Goldblatt R. [9], in the field of dynamic programming methods Salas D.F., Powell W. B. [10].

The present research is devoted to models, methods and algorithms of analytical software design for the organization of interaction of software and information systems. As a tool for their representation a number of meta languages including natural language, meta language of the sets and categories of sets theory were chosen. The structure of models is defined using verbal, mathematical models with different logical restrictions caused by formal graph-analytic high-level programming languages and conditions of their cross-platform implementation in environments of web portals, the intranet, the Internet, etc.

The problem of a synonymy formalization in the task of identification and traceability of an information object at each level of the corresponding description is solved.

Today a particular interest is given to analytical software with elements of intelligent management, capable to generate not only procedural data and reports with predefined structure, but also to provide a possibility of deep analysis by the decision makers.

Thus, it is an urgent task to design and develop the analytical software that can be used by the huge companies in the various business processes, for example in human resource and talent pool management. 
The next problem described in this research is formalization and identification of information objects and maintenance of their compliance to real objects of the studied data domain.

Under information objects the objects of data domain in analytical software causing its structure, attributes, integrity constraints and behavior are understood.

\section{COMPLIANCE AND TRACEABILITY OF REAL AND INFORMATION OBJECTS, PARAMETERS FOR THE KNOWLEDGE BASE AND DESIGN OF ANALYTICAL SOFTWARE}

For definition of real objects, compliance to the correspondent information objects and traceability at the abstract level of the studied educational and production systems the concepts and rules of the ISO/IEC 15288 will be applied.

Let's show that the statements formulated in this standard completely correspond to concepts of Cartesian closed system. As the formal instrument for the description of information objects for modeling, data and knowledge processing and transmission the theory of sets and categories are used.

For further consideration of the mathematical description of real objects and their information processes, let's use the following designations, where:

$F$ is a set of possible states of the analytical software (AS);

$q_{i} \in Q, i=1, \ldots, m$, , where $Q$ is the finite number of the analytical software states;

$v_{i},-$ the information process required for design of the analytical software at a given time;

$V-$ a set of information processes for construction the analytical software.

The transition of the analytical software from one state to another can be described by the function $L$ :

$$
L: F \circ V \rightarrow F
$$

i.e. $l\left(f_{i}, v_{i}\right)$ will display the next state of the analytical software after the execution of the $v_{i}$ construction stage and can be represented by the formula:

$$
f_{i+1}=l\left(f_{i}, v_{i}\right), i=1, \ldots, n .
$$

It is shown that the states of analytical software depend on the states of objects $f_{i}$ and $f_{j}$ given set of morphisms $\operatorname{Hom}\left(f_{i}, f_{j}\right)$ forming a class of objects for each pair (morphisms), for example, $g_{q} \in \operatorname{Hom}\left(f_{i}, f_{j}\right)$ and $l_{q} \in \operatorname{Hom}\left(f_{j}, f_{k}\right)$ where their composition is defined $g_{q} \circ l_{q} \in \operatorname{Hom}\left(f_{j}, f_{k}\right)$. I.e. analytical software states form a category of sets consisting of objects connected by categorical relations.

The problem of analytical software forming for a certain data domain and process of moving the semantics into syntax and in the database takes place (fig. 1).

In figure 2 the concept the Industry 4.0 which is divided into three components is presented: digitalization and integration of virtual value chains, digitalization of products and services, digital business models and access for clients. Carrying out further specification we obtain the set of the following components: mobile devices, cloud computing, augmented reality, personification based on a client profile (multilevel interaction with the client), the analysis of big data, intelligent sensors, 3D - printing, location tracking, the internet of things, cyber security, advanced interaction interfaces between the person and the computer. There are relations between these 11 components.

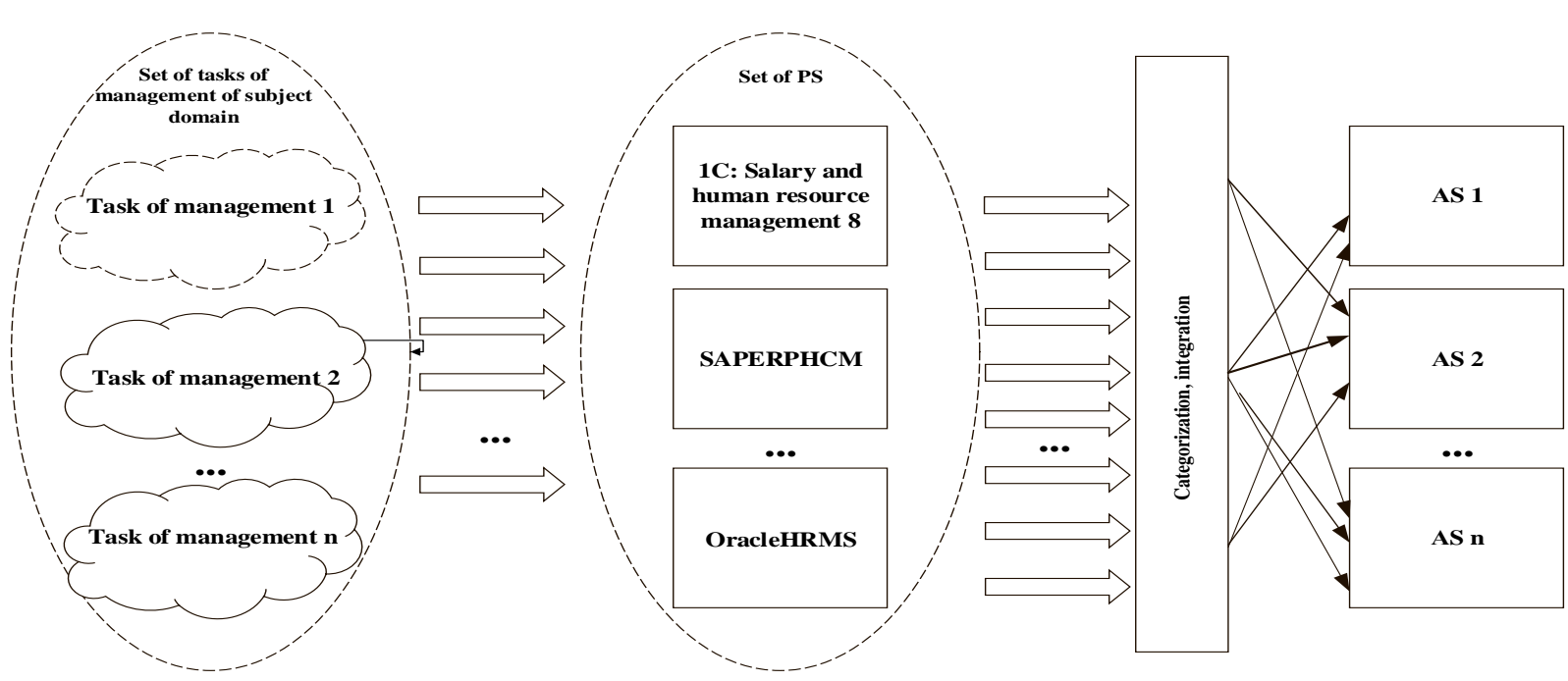

Fig. 1 Display of model of subject domain in the analytical software (AS) 


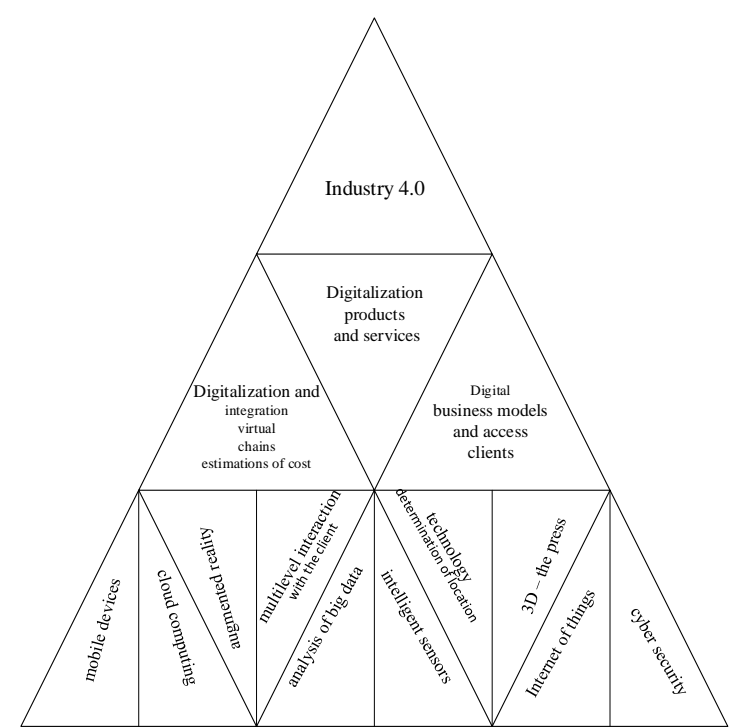

Fig. 2 The main concepts the Industry 4.0

According to the Industry 4.0 concept modern enterprises aim to apply digital production, implementing smart technologies. Management is carried out on the basis of knowledge held by both universities and enterprises, the combination of such knowledge eventually leads to the creation of an educational and production structures.

At the same time, the set of the relations can be considered in the form of additional objects of Big data. Considering evolution of the concept from "the Industry $1.0 "$ to "the Industry $4.0 "$ we can mark out some aspects:

- "The industry 1.0" - development of steam engines, mechanical weaving looms.

- "The industry 2.0" - electric energy, line production, division of labor.

- "The industry 3.0" - automation and the roboequipment.

- "The industry 4.0" - cyberphysical systems, connection of real objects with information processes or virtual objects through information networks and the Internet. Moreover "the Industry 4.0" assumes digitalization and integration of processes down beginning from development (products or purchases) up to production of logistics and service, and integration includes also suppliers and consumers across.

Thus, a virtual object is an object obtained as a result of relations, being completed to a Cartesian closed category, which also does not contradict the Law of requisite variety introduced by W. Ross Ashby [11].

Industrial enterprises have accumulated a sufficiently large amount of data that is heterogeneous and often poorly formalized. So the so-called problem of Big data takes place.

In a broad sense, the term Big data refers to the collection, storage, analysis, transmission, visualization, updating of structured, unstructured and semi-structured data, characterized by a large amount of data [12].

For the representation of knowledge about the subject area, it is necessary to use formalized graphanalytical meta languages, formal logical properties and rules.

The method of constructing Chomsky hierarchies on the basis of the category approach using settheoretical models along with the requirements of systems engineering standards allows us to form boundaries between semantic and syntactic descriptions of the processes of the studied domain and functional modules of the analytical software [13, 14].

At the same time, the formation of the semantic model is shifting to the area of formalizing logical relationships. Applying a systematic approach to determine the boundaries of the studied domain, we determine the goal (semantic measure) and then the point of view. Obviously, there is a delimitation of the subject area. Next, we define the functional content of the subject area (context diagram), and the interaction with the external environment occurs through input, output, control (restrictions) and the mechanism (resources) (Fig. 3).

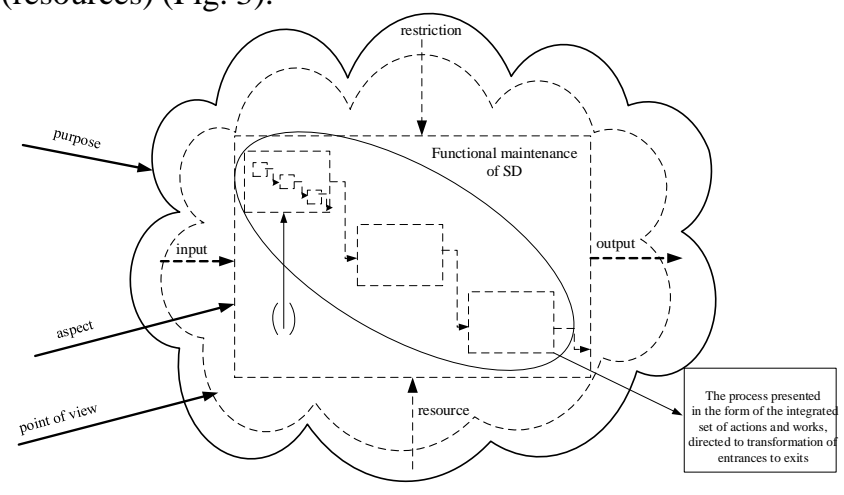

Fig. 3 Delimitation of subject domain (SD)

Let's define "relation" as mathematical structure which formally defines properties of various objects and their interrelation (i.e. as a subset of Cartesian product). Applying a method syntactically - the focused broadcasting, based on N. Chomsky [13] works, we can consolidate the semantic analysis to the syntactic two step procedure: at first - to distinguish structure, then - to construct output actions on its basis. And as a result we come to a mathematical method of the analysis of subject domain [13, 14] (it is based on definition of some final list of chains and their consideration), further we can consider an information system in the form of a final set of functions and the relations between them (i.e. we can consider the relations in the form of functions irrespective of the corresponding objects).

Therefore, the formalized graphic-analytical meta languages which formal logical properties and rules, have to be based on basic provisions of the theory of sets, the theory of categories and N. Chomsky's grammars not above the second level [13] are applied 
to representation of knowledge of the studied subject domain (system modeling).

The method of semantic differentials of $\mathrm{Ch}$. Osgood which shows that the relations between real objects, are connected by the denial relation (contrast according to Descartes) is applied to carrying out quantitative and quality standard of the relations between information objects, and virtual objects are private definitions in terminology of a square of Descartes. [13].

Thus, we can say that the relations of two objects in some cases can be presented in the form of a new object which can be either real, or virtual.

According to classification of formal grammars of N. Chomsky, we can make a classification of systems to 4 levels:

0 - general system (unlimited);

1 - the description of a system using metalanguage (context-dependent);

2 - expands a concept of semantics, showing interaction with the external environment, the system is modelled and created by means of CASE-tools and high-level programming languages (context-free);

3 - completely formalized system which is applied during creation of low-level compilers; the syntax is defined, but semantics is simple, described by the word set and the relationship between these words.

A prototype of analytical software for the processes of HR und talent pool management, consisting of the $1 \mathrm{C}$ information system, the Prognoz Platform and the software tool for interaction of these applications was designed.

The structure of information kernel of intelligent content represents a set of information-analytical systems with the corresponding applications, databases, knowledge bases, etc., it is open as it can constantly be supplemented with new structural and connected content management systems.

The system allows using different modules. There is one more step to increase to the abstraction layer. A part of information which was in semantics is transferred to syntax.

The analytical software is organized using webportal technologies and runs via the web-browser. Data transmission happens in the online-mode. The interface is completely russified (including error messages)

At competent analytical extraction of these data, there is an opportunity to make strategic development plans for the enterprise, to quickly make management decisions. The principle of ensuring "electronic" transparency in organizational and economic, legal and many other aspects for educational production systems is in many respects realized. The possibility of ensuring "electronic" transparency and in administrative and technological and other aspects extends, i.e. such organizations gain properties open or half-open.

The analytical software provides the separation of access rights to the functions of the software and protection against unauthorized access to information in accordance with the requirements of current legislation in the field of information protection $[15$, $16]$.

The analytical software is developed using highlevel programming languages $\mathrm{C \#}$, Python in VisualStudio 2017 [17,18,19,20].

For the developed subsystems, interaction with adjacent and external information systems through file exchange in XML format, as well as calls to external system services via SOAP is provided.

The external data can also be uploaded into the system using XLS and CSV formats.

The fragment of a program code is implemented in the form of the converter which provides data integration of two information systems in order to create analytical reports and panels (fig. 4).

The existing analytical software features can be upgraded in the following way:

- adding of the new features;

- connection of additional services;

- expansion of the list of the data interchange formats with external sources.

The structure and data format of the analytical software are defined by subject domain.

The system controls the correctness of information entered automatically from third-party official information systems, and also verifies the logical integrity of the information in the database when performing any operation.

Users interact with the analytical software using the graphical interface.

All features support Russian language and provide the Russian-speaking user interface meeting all standards and rules of Russian [21,22,23].

Server parts of all subsystems are designed for cross-platform use [24,25,26].

The database management system (DBMS) of all subsystems provides transactional mechanisms, declarative referential integrity, allows you to increase performance by increasing computational power without changes at the application level. General requirements to DBMS:

- relational database model support;

- client-server technology support;

- multiprocessor architecture support;

- availability of tools for indexes and clusters of data;

- automatic recovery of the database;

- availability of a locking gear of transactions;

- availability of the built-in monitoring aids of integrity of databases; 


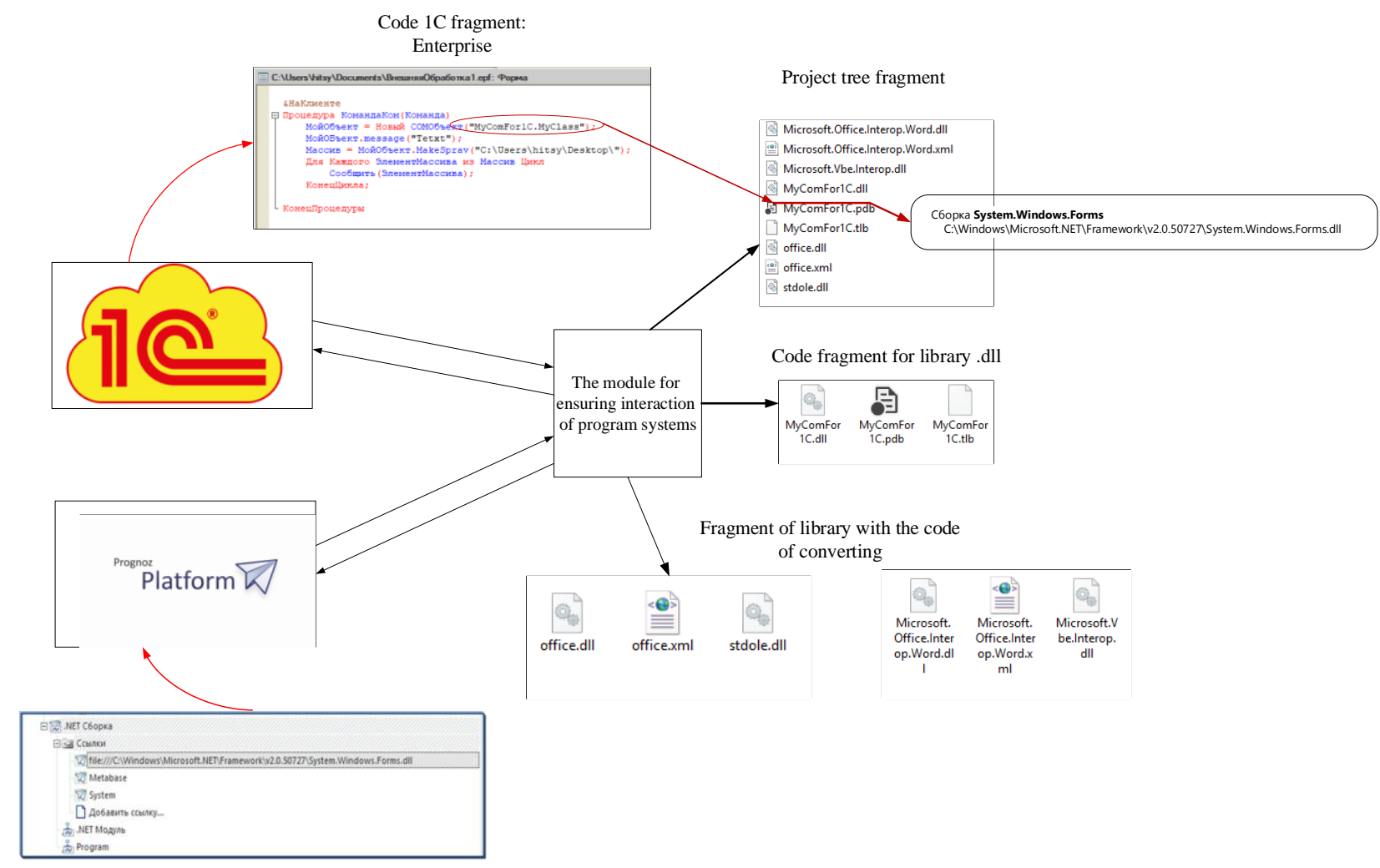

Fig. 4 Example of program realization of analytical software

- availability of the built-in means of database backup;

- support of data import and export (XML, EDIFACT, DBF, TXT, MS of Excel, Word);

- support of TCP/IP protocols;

- availability of graphic administrative tools;

- possibility of data access control;

- centralized user management;

- query tuning;

- availability of the built-in procedures;

- availability of utilities for data transfer from external systems;

- data security provision at the level of the server of databases.

The analytical software will allow to carry out reengineering of program systems, providing expansibility of a system, due to building of additional modules (real objects), to add new functions to a system, to adapt a system (due to adding of aggregated data in the rule base) [25].

The interface of the system provides multiple factor, multiple parameter visualization of data domain and allows supporting the following program functions:

- display of the external environment by means of the specialized multidimensional and multiple-factor graphic interface;

- information representation according to the principles: consolidation (roll-up), drill-down, slicing and dicing;

- expansion of functionality of the software due to migration of technological templates both between levels of an organization structure, and between different systems;

- greatest possible filling of a spatio-temporal fragment of historical, actual and planned content;

- ensuring processing of heterogeneous information and the connectivity of information for all tiers of the internal and external environment;

- interchange by data with programs of third-party developers, etc.

The analytical software can be helpful for information support of decision-making processes providing data collection, monitoring and analysis and metadata concerning the managed processes, for example a talent pool management.

The analytical software automates the following processes:

- introduction and storage of data on all the facts and activities of the organization, quick access to the data;

- display and visualization of the data with use of cross-platform technologies;

- monitoring of the business processes (BPMN models, UML charts, semantic networks, etc.);

- data analysis and display of analytical information (OLAP cubes, analytical panels, tabular display of analytical data, etc.);

- display of media content (content: video, photo, presentations, etc.). 


\section{CONCLUSION}

Virtual objects are the opposite of the concepts of real objects, but their properties intersect, i.e. the properties of real and virtual objects have the same structure and correspond to the functor relations of category theory. As a rule, virtual objects have broader potential properties than real objects. As real and virtual objects can be displayed in a symbolical form in information systems, virtual objects can be replaced by real objects while maintaining the properties of the virtual object. Note also that not all properties of virtual objects can be implemented in the new real object, so the new virtual object with the remaining unrealized properties is implemented in the information system. It can be shown that the relations between real objects are connected by the negation relation (the opposite of Descartes), and virtual objects are particular definitions in the terminology of the Descartes square.

\section{REFERENCES}

[1]. Kovalyov, S.P. Teoretiko-kategorny approach to design of program systems. Base. and applied mathematics, 19, 2014, ss. 111-170

[2]. Massel L.V. Fractal approach to structuring knowledge and examples of its application. Design ontology, 2016, 6 (2) pp. 149161

[3]. Kulikov G.G., Beltyukov A.P., Nabatov A.N., Shamidanov D.G. A method of formation of a metastructure of content of subject domain in the form of the local distributed information system. Collection of the Sixth International scientific conference, 2017, Page 144-148. (in Russian)

[4]. Antonov V.V. A design method of an adaptive program complex on the basis of methodology of category formal model of open subject domain. UGATU bulletin, 2015, 19 (1) pp. 258-263 (in Russian)

[5]. Kulikov G.G., Antonov V.V., Fakhrullina A.R., Rodionova L.E., Formal representation of model of realization of functions of system engineering on the basis of the principle of a necessary variety of structural communications. Messenger SUSU. "Computer Technologies, Management, Radio Electronics" series, 17(4), 2017, pp. 146-153 (in Russian)

[6]. Martynov V.V., Filosova E.I., Shiryaev O.V. The organization of preparation and information podderzhkarealization of the dynamic educational programs considering requirements of the employer. Collection of scientific works of the Fourteenth international conference "Management of economy: methods, models, technologies", 2014, 1(1) pp.110-113 (in Russian)

[7]. Mironov, V.V., Yusupova N.I., Shakirova G. R. The situational oriented databases: concept, architecture, XML-implementation UGATU Bulletin, 2 (37), 2011, pp. 233-244

[8]. Kovács, G., Yussupova, N., Rizvanov, D. Resource management simulation using multi-agent approach and semantic constraints. An International Journal for Engineering and Information Sciences, 12 (1), 2017, pp. 45-58

[9]. Goldblattr. Top wasps. Kategorny analysis of logic, 1983, Page 486

[10]. The Big Data Conundrum: How to Define It? [Electronic resource] // MIT Technology Review [Official website]. 2013.
[11]. Kulikov G.G., V.V. Antonov, D.V. Antonovteoreticheskiye and, applied aspects of creation of models of information systems. LAP LAMBERT Academic Publishing GmbH\& Co.KG, Germany, 2011, 134 pages.

[12]. Feoktistov A., Gorsky S., Sidorov I., Kostromin R., Edelev A., Massel L., Energy Research Application Development Through Convergence of Grid and Cloud Computing //Communications in Computer and Information Science/ Supercomputing, 2019 (in Russian)

[13]. Chomsky N. Language and problem of knowledge MSU Bulletin, 1996; 157-185

[14]. Osgood Ch., Sushi Dzh., Tannenbaum P. Application of a technique semantic дифференциалак to researches on an esthetics and adjacent problems. Semiotics and iskusstvometriya, 1972, pp. $46-48$.

[15]. Vorobev, S., Edelev, A.: Analysis of the importance of critical objects of the gas industry with the method of determining critical elements in networks of technical infrastructures. In: 10th International Conference on Management of Large-Scale System Development (MLSD). IEEE 2017.

[16]. Voevodin, V.V.: The solution of large problems in distributed computational media. Automat. Remote Control 68(5)

[17]. Qin, H., Zhu, L.: Subject oriented autonomic cloud data center networks model. J. Data Anal. Inform. Process, 2017, 5(3), pp.87-95

[18]. Sokolinsky, L.B., Shamakina, A.V.: Methods of resource management in problem-oriented computing environment. Program. Comput. Soft, 2016, 42(1), pp.17-26

[19]. Voropaj N.I., Massel' L.V., Slavin G.B. Setting up the system for monitoring the power engineering facilities in Russia using new information technologies // V. 4, № 1, P. 119-137 (2018) (in Russian)

[20]. Kutluyarov R. V., Bagmanov V. Kh., V. V. Antonov, Sultanov A. K. and Lyubopytov V. S., "Increase of nonlinear signal distortions due to linear mode coupling in space division multiplexed systems," 2017 International Multi-Conference on Engineering, Computer and Information Sciences (SIBIRCON), Novosibirsk, 2017, pp. 282-286. (in Russian)

[21]. Kutluyarov R.V., Lyubopytovab V.S., Bagmanov V.Kh. Sultanov A. Kh. Influence of the linear mode coupling on the nonlinear impairments in few-mode fibers // Procedia Engineering. - 2017 P. 148-154 (in Russian)

[22]. Ho K.P., Kahn J.M. Mode coupling and its Impact on spatially multiplexed systems, in: I.P. Kaminow, T. Li and A.E. Willner (Eds.), Optical Fiber Telecommunications VIB: Systems and Networks, Elsevier, San Diego 2013

[23]. Yusupova N., Smetanina O., Agadullina A. and Rassadnikova E., "The development of ontologies to support the decisions in production systems management," 2017 Second Russia and Pacific Conference on Computer Technology and Applications (RPC), Vladivostok, 2017, pp. 188-193.

[24]. Sokolinsky, L.B., Shamakina, A.V.: Methods of resource management in problem-oriented computing environment. Program. Comput. Soft. 42(1), pp.17-26 2016

[25]. Massel, L.V., Arshinsky, V.L., Massel, A.G.: Intelligent computing on the basis of cognitive and event modeling and its application in energy security studies. Int. J. Energy Optim. Eng. 3(1), pp.83-91 2014 (in Russian)

[26]. Salas D.F., Powell W.B. Benchmarking a scalable approximate dynamic programming algorithm for stochastic control of grid-level energy storage. INFORMS Journal on Computing: 30(1), 2018, pp.106-123. 